PROCEEDINGS OF THE

AMERICAN MATHEMATICAL SOCIETY

Volume 124, Number 12, December 1996, Pages 3885-3888

S 0002-9939(96)03268-6

\title{
A COEFFICIENT OF AN ASYMPTOTIC EXPANSION OF LOGARITHMS OF DETERMINANTS FOR CLASSICAL ELLIPTIC PSEUDODIFFERENTIAL OPERATORS WITH PARAMETERS
}

\author{
YOONWEON LEE
}

(Communicated by Peter Li)

\begin{abstract}
For classical elliptic pseudodifferential operators $A(\lambda)$ of order $m>0$ with parameter $\lambda$ of weight $\chi>0$, it is known that $\log \operatorname{det}_{\theta} A(\lambda)$ admits an asymptotic expansion as $\lambda \rightarrow+\infty$. In this paper we show, with some assumptions, that the coefficient of $\lambda^{-1 / \chi}$ can be expressed by the value of a zeta function at 0 for some elliptic $\psi \mathrm{DO}$ on $M \times S^{1}$ multiplied by $\frac{m}{2}$.
\end{abstract}

\section{INTRODUCTION}

Let $M$ be a compact oriented Riemannian manifold of dimension $d$ and let $E \stackrel{\pi}{\rightarrow}$ $\rightarrow M$ be a vector bundle of rank $k$. Let $A(\lambda): C^{\infty}(E) \rightarrow C^{\infty}(E)$ be a classical elliptic pseudodifferential operator of order $m>0$ with parameter $\lambda$ of weight $\chi>0$, where $\lambda$ is a nonnegative real number. That is, the symbol of $A(\lambda)$ has an asymptotic expansion as in (2.1).

We assume that there is an angle $\theta$ such that the principal symbol $a_{m}(x, \xi, \lambda)$ does not have any eigenvalues on the ray $\left\{z \in \mathbf{C} \mid z=\rho e^{i \theta}, \rho \geq 0\right\}$ for $|\xi|+|\lambda|^{\frac{1}{\chi}} \neq 0$ and that $A(\lambda)$ does not have any eigenvalues in a sector $L_{[\theta-\varepsilon, \theta+\varepsilon]}=\{z \in \mathbf{C} \mid \theta-\varepsilon \leq$ $\arg z \leq \theta+\varepsilon\}$ for some small $\varepsilon>0$. We call this $\theta$ an Agmon angle. In fact from the compactness of the set $\left\{\left.(x, \xi, \lambda)|x \in M,| \xi\right|^{2}+|\lambda|^{\frac{2}{\chi}}=1\right\}$, we know that $a_{m}(x, \xi, \lambda)$ does not have any eigenvalues in a sector $L_{[\theta-\delta, \theta+\delta]}$ for sufficiently small $\delta>0$.

It is shown in $[\mathrm{BFK}]$ that as $\lambda \rightarrow+\infty, \log \operatorname{det}_{\theta} A(\lambda)$ admits an asymptotic expansion of the form

$$
\log \operatorname{det}_{\theta} A(\lambda) \sim \pi_{-d} \lambda^{\frac{d}{\chi}}+\pi_{-d+1} \lambda^{\frac{d-1}{\chi}}+\cdots+\pi_{0}+\pi_{1} \lambda^{-\frac{1}{\chi}}+\cdots+\sum_{j=0}^{d} q_{j} \lambda^{\frac{j}{\chi}} \log \lambda
$$

as $\lambda \rightarrow+\infty$. Here each coefficient $\pi_{i}$ and $q_{j}$ is computable in terms of the asymptotic symbol of $A(\lambda)$.

Let $\sum_{j=0}^{\infty} a_{m-j}(x, \xi, \lambda)$ be an asymptotic symbol of $A(\lambda)$ for some local coordinate $U$. We also assume that for each $j$, the function $\tilde{a}_{m-j}: U \times \mathbf{R}^{d} \times \mathbf{R} \rightarrow$ $\{k \times k$ matrices $\}$ defined by $\tilde{a}_{m-j}(x, \xi, \lambda)=a_{m-j}\left(x, \xi,|\lambda|^{\chi}\right)$ is smooth. Then from $A(\lambda)$ we can construct a classical elliptic pseudodifferential operator $P$ with the

Received by the editors September 6, 1994 and, in revised form, December 12, 1994.

1991 Mathematics Subject Classification. Primary 58G15, 58G26.

(C)1996 American Mathematical Society 
same Agmon angle $\theta$ on $M \times S^{1}$, where $P$ is uniquely determined up to smoothing operators. Let $\zeta_{P}(s)$ be the zeta function constructed from the eigenvalues of $P$.

The purpose of this note is to show that $\pi_{1}=\frac{m}{2} \zeta_{P}(0)$. So far we are unable to give a similar interpretation of other coefficients $\pi_{i}(i \neq 1)$ and $q_{j}$.

\section{Construction of $P$}

Suppose that in a local coordinate system $U$, the asymptotic symbol of $A(\lambda)$ is $\sigma(A(\lambda)) \sim \sum_{j=0}^{\infty} a_{m-j}(x, \xi, \lambda)$. Then

$$
a_{m-j}(x, \xi, \lambda): U \times \mathbf{R}^{d} \times[0, \infty) \rightarrow\{k \times k \text { matrices }\}
$$

with

$$
a_{m-j}\left(x, t \xi, t^{\chi} \lambda\right)=t^{m-j} a_{m-j}(x, \xi, \lambda) \quad \text { for } t>0 .
$$

Now for each $j$, we extend $a_{m-j}$ to

$$
\begin{gathered}
\tilde{a}_{m-j}: U \times S^{1} \times \mathbf{R}^{d} \times \mathbf{R} \rightarrow\{k \times k \text { matrices }\} \text { by } \\
(x, t, \xi, \lambda) \mapsto a_{m-j}\left(x, \xi,|\lambda|^{\chi}\right) .
\end{gathered}
$$

Then $\tilde{a}_{m-j}$ is smooth by the assumption of the smoothness of $a_{m-j}\left(x, \xi,|\lambda|^{\chi}\right)$. Note that $\tilde{a}_{m-j}(x, t, \xi, \lambda)$ is a homogeneous function of degree $m-j$ with respect to $\xi, \lambda$.

Consider a diagram

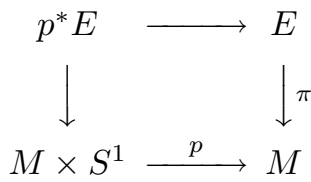

where $p$ is the natural projection.

Choose an elliptic $\psi$ DO $\widetilde{A}: C^{\infty}\left(p^{*} E\right) \rightarrow C^{\infty}\left(p^{*} E\right)$ whose asymptotic symbol is $\sigma(\widetilde{A}) \sim \sum_{j=0}^{\infty} \tilde{a}_{m-j}(x, t, \xi, \lambda)$ in a local coordinate $U \times S^{1}$. Since the principal symbol $a_{m}\left(x, \xi,|\lambda|^{\chi}\right)$ of $\widetilde{A}$ does not have any eigenvalues in the sector $L_{[\theta-\delta, \theta+\delta]}$ for $|\xi|+|\lambda|^{\frac{1}{\chi}} \neq 0$, there exists $R>0$ such that $\operatorname{Spectrum}(\widetilde{A}) \cap\{z|| z \mid \geq R, \theta-\delta \leq \arg z \leq$ $\theta+\delta\}$ is empty (see [Sh] for details). Since the spectrum of $\widetilde{A}$ is discrete, there are only finitely many eigenvalues of $\widetilde{A}$ in $\{z|| z \mid<R, \theta-\delta \leq \arg z \leq \theta+\delta\}$. Note that each eigenspace corresponding to an eigenvalue is a finite-dimensional vector space by the ellipticity of $\widetilde{A}$ of order $m>0$. Let $Q$ be the span of \{eigenvectors of $\widetilde{A}$ whose eigenvalues are $\rho e^{i \theta}$ for some $\left.\rho, 0 \leq \rho \leq R\right\}$. Then $Q$ is a finite-dimensional vector space. Define $\phi: C^{\infty}\left(p^{*} E\right) \rightarrow C^{\infty}\left(p^{*} E\right)$ be the natural projection onto $Q$.

Define $P=\widetilde{A}-R e^{i \theta} \phi$. Then $P$ is injective with an Agmon angle $\theta$ and the asymptotic symbol of $P$ is exactly the same as the asymptotic symbol of $\widetilde{A}$. Define $\zeta_{P}(s)=\sum_{\lambda_{i}} \lambda_{i}^{-s}$, where $\lambda_{i}$ 's are the eigenvalues of $P$. Then by [Se] (also see [Wo]), $\zeta_{P}(s)$ is regular at 0 with

$$
\begin{aligned}
\zeta_{P}(0)= & \frac{e^{i \theta}}{m(2 \pi)^{d+1}} \\
& \cdot \int_{M \times S^{1}} d \operatorname{vol}(x, t) \int_{|\xi|^{2}+\lambda^{2}=1} d(\xi, \lambda)^{\prime} \int_{0}^{\infty} \operatorname{tr} \tilde{r}_{-m-d-1}\left(x, t, \xi, \lambda, e^{i \theta} \mu\right) d \mu,
\end{aligned}
$$


where $\tilde{r}_{-m-d-1}$ is the homogeneous part of degree $-m-d-1$ in the asymptotic symbol of the resolvent $(P-\mu I)^{-1}$. Note that $\tilde{r}_{-m-d-1}\left(x, t, \xi, \lambda, e^{i \theta} \mu\right)$ does not depend on $t$ in this case.

Recall that $\log \operatorname{det}_{\theta} A(\lambda) \sim \pi_{-d} \lambda^{\frac{d}{x}}+\cdots+\pi_{0}+\pi_{1} \lambda^{-\frac{1}{x}}+\cdots+\sum_{j=0}^{d} q_{j} \lambda^{\frac{j}{x}} \log \lambda$ as $\lambda \rightarrow+\infty$. Then our goal is to prove the following theorem.

Theorem. $\pi_{1}=\frac{m}{2} \zeta_{P}(0)$.

\section{Proof of the Theorem}

From the Appendix of $[\mathrm{BFK}]$ we can derive the local formula

$$
\pi_{1}=\frac{-e^{i \theta}}{(2 \pi)^{d}} \int_{M} \int_{\mathbf{R}^{d}} \int_{0}^{\infty} \operatorname{tr} r_{-m-d-1}\left(x, \xi, 1, e^{i \theta} \mu\right) d \mu d \xi d \operatorname{vol}(x),
$$

where $r_{-m-d-1}$ is the homogeneous part of degree $-m-d-1$ in the asymptotic symbol of the resolvent $(A(\lambda)-\mu I)^{-1}$.

Note the relation $\tilde{r}_{-m-d-1}\left(x, t, \xi, \lambda, e^{i \theta} \mu\right)=r_{-m-d-1}\left(x, \xi,|\lambda|^{\chi}, e^{i \theta} \mu\right)$. Then

$$
\begin{aligned}
\zeta_{P}(0)= & -\frac{2 e^{i \theta} \cdot 2 \pi}{m(2 \pi)^{d+1}} \\
& \cdot \int_{M} d \operatorname{vol}(x) \int_{\substack{|\xi|^{2}+\lambda^{2}=1 \\
\lambda>0}} d(\xi, \lambda)^{\prime} \int_{0}^{\infty} \operatorname{tr} r_{-m-d-1}\left(x, \xi,|\lambda|^{\chi}, e^{i \theta} \mu\right) d \mu
\end{aligned}
$$

since the integrand is even in $\lambda$.

Set

$$
(\mathrm{I})=\int_{\substack{|\xi|^{2}+\lambda^{2}=1 \\ \lambda>0}} d(\xi, \lambda)^{\prime} \int_{0}^{\infty} \operatorname{tr} r_{-m-d-1}\left(x, \xi,|\lambda|^{\chi}, e^{i \theta} \mu\right) d \mu
$$

Then using the projection from the upper hemisphere to $\left\{\xi \in \mathbf{R}^{d} \| \xi \mid<1\right\}$, we obtain

$$
\begin{aligned}
(\mathrm{I})= & \int_{|\xi|<1} \int_{0}^{\infty} \operatorname{tr} r_{-m-d-1}\left(x, \xi,\left(\sqrt{1-|\xi|^{2}}\right)^{\chi}, e^{i \theta} \mu\right)\left(1-|\xi|^{2}\right)^{-\frac{1}{2}} d \mu d \xi \\
= & \int_{|\xi|<1} \int_{0}^{\infty}\left(1-|\xi|^{2}\right)^{-\frac{m+d+2}{2}} \operatorname{tr} r_{-m-d-1} \\
& \cdot\left(x, \frac{\xi_{1}}{\sqrt{1-|\xi|^{2}}}, \ldots, \frac{\xi_{d}}{\sqrt{1-|\xi|^{2}}}, 1, e^{i \theta} \frac{\mu}{{\sqrt{1-|\xi|^{2}}}^{m}}\right) d \mu d \xi,
\end{aligned}
$$

since the weight of $\mu$ is $m$.

Consider a map $\Phi: \mathbf{R}^{d} \times(0, \infty) \rightarrow\left\{\xi \in \mathbf{R}^{d}|| \xi \mid<1\right\} \times(0, \infty)$ defined by

$$
\left(\eta_{1}, \ldots, \eta_{d}, \nu\right) \mapsto\left(\frac{\eta_{1}}{\sqrt{1+|\eta|^{2}}}, \ldots, \frac{\eta_{d}}{\sqrt{1+|\eta|^{2}}}, \frac{\nu}{{\sqrt{1+|\eta|^{2}}}^{m}}\right)=\left(\xi_{1}, \ldots, \xi_{d}, \mu\right) .
$$

Then $\Phi$ is a diffeomorphism with $\operatorname{det}(J(\Phi))=\left(1+|\eta|^{2}\right)^{-\frac{m+d+2}{2}}=\left(1-|\xi|^{2}\right)^{\frac{m+d+2}{2}}$. Hence we can get $(\mathrm{I})=\int_{\mathbf{R}^{d}} \int_{0}^{\infty} \operatorname{tr} r_{-m-d-1}\left(x, \eta, 1, e^{i \theta} \nu\right) d \nu d \eta$.

\section{ACKNOWLEDGMent}

I am very grateful to D. Burghelea and T. Kappeler for their valuable advice on this problem and to the referee for his suggestion for the shorter proof. 


\section{REFERENCES}

[BFK] D. Burghelea, L. Friedlander, and T. Kappeler, Mayer-Vietoris type formula for determinants of elliptic differential operators, J. Funct. Anal. 107 (1992), 34-65. MR 93f:58242

[Se] R. Seeley, Complex powers of an elliptic operator, Proc. Sympos. Pure Math., vol. 10, Amer. Math. Soc., Providence, RI, 1967, pp. 288-307. MR 38:6220

[Sh] M. A. Shubin, Pseudodifferential operators and spectral theory, Springer-Verlag, Berlin and New York, 1985.

[Wo] M. Wodzicki, Spectral asymmetry and zeta functions, Invent. Math. 66 (1982), 115-135. MR 83h: 58097

Department of Mathematics, Ohio State University, Columbus, Ohio 43210

Current address: Department of Mathematics, College of Natural Science, Inha University, 253 Yonghyun-dong, Nam-gu, Inchon, Korea 402-751

E-mail address: ywonlee@dragon.inha.ac.kr 